\title{
General models of Einstein gravity with a non-Newtonian weak-field limit
}

\author{
Mariano Cadoni*and Marcello Casula \\ Dipartimento di Fisica, Università di Cagliari and INFN, Sezione di Cagliari \\ Cittadella Universitaria, 09042 Monserrato, Italy
}

December 14, 2018

\begin{abstract}
We investigate Einstein theories of gravity, coupled to a scalar field $\varphi$ and point-like matter, which are characterized by a scalar field-dependent matter coupling function $e^{H(\varphi)}$. We show that under mild constraints on the form of the potential for the scalar field, there are a broad class of Einstein-like gravity models -characterized by the asymptotic behavior of $H$ - which allow for a non-Newtonian weak-field limit with the gravitational potential behaving for large distances as $\ln r$. The Newtonian term $G M / r$ appears only as sub-leading. We point out that this behavior is also shared by gravity models described by $f(R)$ Lagrangians. The relevance of our results for the building of infrared modified theories of gravity and for modified Newtonian dynamics is also discussed.
\end{abstract}

\section{Introduction}

In the last decade there has been growing interest for gravitational models with largedistance deviations from standard, Einsteinian and Newtonian gravity [1, 2, 3, 4, 5, 6, 17, 8, 9, 10. The main reason behind this interest is the hope that the accelerated expansion of the universe and rotational curves of the galaxies could be explained by large distance modifications of our present understanding of gravity and inertia, without postulating nonbaryonic forms of matter such as dark matter and dark energy [11, 12]. One of the most promising possibilities is represented, in the context of the brane-world scenario, by the Dvali-Garbadze-Porrati (DGP) model [1]. In the DGP model the gravitational interaction is described by five-dimensional Einstein gravity and the usual four-dimensional (4D) Newtonian potential is generated by a $4 \mathrm{D}$ scalar curvature term in the world-volume brane action.

The idea of modifying Newton's law and/or Einstein gravity at large distances is not new. Since galactic rotational curves were found to be inconsistent with the distribution of luminous matter, such alternative theories of gravity have been proposed and pushed forward [3, 4, 6]. On the other hand, from the experimental side, there is plenty of room for such infrared modifications of gravity; our experimental knowledge of gravity conforms with general relativity but is limited to distances between say $10^{-3} \mathrm{~cm}-100 \mathrm{MPc}$.

*email: mariano.cadoni@ca.infn.it 
A quite generic prediction of infrared-modified theories of gravity is the presence of large-distance logarithmic corrections to the Newtonian potential. In the DGP model, a $\ln (r / L)$ term, where $L$ is a cross-over length, appears as correction of the Newtonian potential [1, 13]. In models that modify Newtonian dynamics to account for galactic rotational curves the logarithmic corrections dominates for small accelerations, of order $a_{0} \sim 10^{-29} \mathrm{~cm}^{-1}$ (in natural units) [3, 4].

The search for Einstein-like theories of gravity that allow for a non-Newtonian weakfield limit with large-distance logarithmic correction is therefore of great interest. Such models can be considered as an effective descriptions of more general theories of gravity, such as those emerging in the brane-world scenario, which modify the gravitational interaction at large distance.

It is not easy to build metric, Einstein-like, theories of gravity that allow for a nonNewtonian weak-field limit. Typically one has to introduce additional fields (scalars or vectors) and add to the Einstein-Hilbert action additional terms. Alternatively, one may consider higher-curvature terms in the gravitational action, e.g. in the form of $f(R)$ theories. In both cases the theory is highly constrained by several phenomenological requirements: existence of a Newtonian potential term at short distances, absence of a fifth force, correct predictions for light bending and so on (see e.g. Ref. [6]).

A simple theory of Einstein gravity coupled to a scalar field with an exponential potential that allows for a $\ln (r / L)$ weak field limit has been proposed in Ref. [14]. The proposed gravity theory requires a particular form of both the scalar field potential and the coupling function between scalar field and matter.

In this paper we show that the existence of a $\ln (r / L)$ weak-field limit is not restricted to the particular model of Ref. [14, but is a quite generic feature of a broad class of models of gravity coupled with a scalar field. We require that the model satisfies the following conditions:

a) Existence of a consistent non-relativistic, static, weak-field limit of the gravitational field equations.

b) At large distance $(r \gg L)$ the gravitational potential is dominated by the $\ln (r / L)$ term.

c) Presence of a sub-leading Newtonian term $-G M / r$.

d) The short distance deviations from Newtonian behavior are at most of order $r^{-\gamma}$ with $\gamma \geq 2$.

We show that virtually all models of Einstein gravity coupled with a scalar field satisfies the above conditions if an appropriate coupling function between the scalar field and matter is introduced. As an illustration of our procedure we discuss in detail the case of a scalar field with a power-law potential. The models can be also rewritten, in the Jordan frame, as a scalar-tensor theory of gravity and also reformulated as a theory of gravity with an $f(R)$ Lagrangian.

The structure of the paper is as follows. In section 2 we present our model. In section 3 we discuss the weak-field limit. In section 4 we derive the general solution. In section 5 we illustrate our general procedure for the particular case of a scalar field with a power-law potential. The relevance of our models for describing the infrared modifications of the gravitational interaction is discussed in section 6 . In section 7 we formulate our model in the Jordan frame and we stress its equivalence with a $f(R)$ theory of gravity. Finally, in section 8 we state our conclusions. 


\section{The model}

Let us consider a system of two point-particles of mass $M$ (the source) and $m$ (test particle), with $M \gg m$ interacting with the gravitational field $g_{\mu \nu}$ and a scalar field $\varphi$. The gravitational field is described by the Einstein-Hilbert action. The scalar field is self-interacting, with potential $V(\varphi, L)$, and its interaction with the point-particles is characterized by a coupling function $e^{H(\varphi, L)}$. Both the potential and the coupling function depend not only on $\varphi$ but also on a real parameter $L$ with dimensions of a length (we use natural units). For a generic, $V(\varphi) \neq \lambda \varphi^{4}$, potential the presence of such a parameter is necessary for purely dimensional reasons, because the scalar field $\varphi$ has dimension of a (length $)^{-1}$. We will show later in detail that this parameter has the physical meaning of the length-scale, above which the non-Newtonian behavior of our gravitational model become relevant.

The classical dynamics of the system is described by the Einstein-like action

$$
S=\int d^{4} x \sqrt{-g}\left[\frac{1}{16 \pi G} R-\partial_{\nu} \varphi \partial^{\nu} \varphi-V\right]-e^{H}\left(M \int d s_{(M)}+m \int d s_{(m)}\right),
$$

where $d s_{(M, m)}$ are respectively the line elements of the source and test particle $d s_{(M, m)}=$ $d t\left[\left(d x_{(M, m)}^{\mu} / d t\right)\left(d x_{(M, m)}^{\nu} / d t\right) g_{\mu \nu}\right]^{1 / 2}$ and we are using for the metric a signature $(-1,1,1,1)$.

In the limit $M \gg m$ the contribution of the test particle to the stress-energy tensor and to the equation for the scalar field can be neglected. Moreover, we are only interested in the motion of the test particle. The resulting equation of motion stemming from the action (11) are,

$$
\begin{aligned}
& R_{\mu \nu}-\frac{1}{2} g_{\mu \nu} R=8 \pi G\left[T_{\mu \nu}^{(\varphi)}+e^{H} T_{\mu \nu}^{(M)}\right] \\
& 2 \nabla^{2} \varphi-\frac{\partial V}{\partial \varphi}=M H^{\prime} e^{H} \int d \tau \frac{\delta^{4}\left(x^{\nu}-x_{(M)}^{\nu}(\tau)\right)}{\sqrt{-g}} \\
& \frac{d^{2} x_{(m)}{ }^{\mu}}{d \tau^{2}}+\tilde{\Gamma}_{\rho \sigma}^{\mu} \frac{d x_{(m)} \rho}{d \tau} \frac{d x_{(m)} \sigma}{d \tau}=0,
\end{aligned}
$$

where the prime denotes derivation with respect to $\varphi$ and $T_{\mu \nu}^{(\varphi)}, T_{\mu \nu}^{(M)}$ are the stressenergy tensors for the scalar field and for the source of mass $M$,respectively, and $\tilde{\Gamma}$ is a $\varphi$-dependent connection:

$$
\begin{aligned}
T_{\mu \nu}^{(\varphi)} & =2 \partial_{\mu} \varphi \partial_{\nu} \varphi-g_{\mu \nu}\left[(\partial \varphi)^{2}+V\right] \\
T_{\mu \nu}^{(M)} & =M \int d \tau u_{\mu} u_{\nu} \frac{\delta^{4}\left(x^{\alpha}-x_{(M)}^{\alpha}(\tau)\right)}{\sqrt{-g}} \\
\tilde{\Gamma}_{\rho \sigma}^{\mu} & =\Gamma_{\rho \sigma}^{\mu}+\frac{1}{2}\left(\partial_{\rho} H \delta_{\sigma}^{\mu}+\partial_{\sigma} H \delta_{\rho}^{\mu}-2 g_{\rho \sigma} g^{\mu \gamma} \partial_{\gamma} H\right) .
\end{aligned}
$$

In the previous equations $u_{\mu}$ is the four-velocity of the source.

\section{The weak-field limit}

We are interested in the usual weak-field, non-relativistic, static limit of the field equations (2). We expand the metric near a flat background, $g_{\mu \nu}=\eta_{\mu \nu}+h_{\mu \nu}$ with $h_{\mu \nu} \ll 1$ and we 
consider field configurations depending only on the spatial coordinates $x^{i}, i=1,2,3$. In the non-relativistic limit, when the velocity of the particles are $\ll 1$ and $\left|T_{i j}\right| \ll\left|T_{00}\right|$, the field equations (2) give,

$$
\begin{aligned}
\bar{\nabla}^{2} \Phi & =4 \pi G\left[(\bar{\nabla} \varphi)^{2}+V\right]+H^{\prime \prime}(\nabla \varphi)^{2}+\frac{1}{2} H^{\prime} V^{\prime}+e^{H}\left(4 \pi G+\frac{H^{\prime 2}}{2}\right) \tilde{T}_{00}^{(M)} \\
\bar{\nabla}^{2} \varphi & =\frac{1}{2} V^{\prime}+\frac{1}{2} e^{H} H^{\prime} \tilde{T}_{00}^{(M)} \\
\frac{d^{2} \bar{x}_{(m)}}{d t^{2}} & =-\bar{\nabla} \Phi,
\end{aligned}
$$

where $\Phi=-h_{00} / 2+H, \tilde{T}_{00}^{(M)}=M \delta^{3}\left(\bar{x}-\bar{x}_{(M)}\right)$. The bar indicates three-dimensional vectorial quantities, and the differential operators are calculated with respect to the threedimensional Euclidean metric.

From the third Equation in (44) it is evident that in the weak-field limit the field $\Phi$ represents the potential that determines the force acting on the test particle. The usual weak-field Newtonian limit can be trivially recovered setting in Eqs. (4) $H=V=0$ and picking the $\varphi=0$ solution for the scalar field equation. In the following we will only consider spherical symmetric solution to the Eqs. (4). They can be found placing the source particle of mass $M$ at the origin of the coordinate system and using spherical coordinates $(r, \theta, \omega)$.

\section{The general solution}

Our goal is to find a general class of models described by the action (11) and parametrized by a particular, albeit general, from of the functions $V(\varphi, L), H(\varphi, L)$, whose solutions satisfy the following conditions:

a) Existence of a consistent non-relativistic, static, weak field limit given by Eq. (4).

b) At large distance $(r \gg L)$ the solutions for $\Phi$ are dominated by a $\ln (r / L)$ term .

c) They must allow for a sub-leading Newtonian term $-G M / r$.

d) The short distance deviations of $\Phi$ from the Newtonian behavior are at most of order $r^{-\gamma}$ with $\gamma \geq 2$.

Obviously, these conditions constraint the form of the scalar potential $V$ and on the coupling function $H$. Consistency of the non-relativistic approximation requires that the stress-energy tensor satisfies the following condition: $\left|T_{i j}\right| \ll\left|T_{00}\right|$. In the case of the source of the gravitational field the previous conditions are satisfied if one considers a point particle moving with speed much lesser then the speed of light. On the other hand for the stress-energy tensor of the scalar field in Eq. (3) we have:

$$
T_{00}^{(\varphi)}=\left(\partial_{r} \varphi\right)^{2}+V, \quad T_{\mu}^{(\varphi) \mu}=-4 V-2\left(\partial_{r} \varphi\right)^{2} .
$$

It follows that the conditions for the validity of the non relativistic approximation are consistent with the weak-field equations (4) only if $V$ and $\left(\partial_{r} \varphi\right)^{2}$ do not affect the Newtonian term in the potential $\Phi$. This together with condition $d$ ) above imply the large $r$, $r \gg L$ behavior:

$$
V(\varphi) \sim\left(\partial_{r} \varphi\right)^{2} \sim \mathcal{O}\left(r^{-\beta}\right), \quad \beta \geq 4
$$


Condition $c$ ) requires the presence of the term $-M G / r$ in the solution for $\Phi$. In view of the first equation in (4) this implies $\left.\left(4 \pi G+H^{\prime 2}\right) e^{H}\right|_{r=0}=4 \pi G$. The simplest solution to this equation is given by

$$
H(r=0)=H^{\prime}(r=0)=0 .
$$

Our next and last task is to fulfil condition $b$ ). Using (7) and the equations of motion for the scalar field $\varphi$, the first equation in (4) becomes

$$
\bar{\nabla}^{2} \Phi=\bar{\nabla}^{2} H+4 \pi G T_{00}^{(M)} .
$$

In order to have a solution for the gravitational potential $\Phi$ satisfying at the same time condition $d$ ), we must have for $r \gg L$

$$
\bar{\nabla}^{2} H=\frac{C}{r^{2}}+\mathcal{O}\left(r^{-4}\right)
$$

where $\mathrm{C}$ is an arbitrary constant. Obviously Eq. (9) determines the dependence of $H$ on $r$. In order to find the dependence of the coupling function $H$ on $\varphi$, we need to solve first the equation of motion for $\varphi(r)$. The $r \gg L$ asymptotic form of the solution of Eq. (9) is

$$
H=C \ln \frac{r}{L}+C_{1}+\mathcal{O}\left(r^{-2}\right),
$$

where $C_{1}$ is an arbitrary constant. Notice that in principle we can use Eq. (8) to describe an arbitrary deviation (not necessarily logarithmic) of the gravitational potential from its Newtonian behavior. Obviously in this case Eq. (9) as to be modified accordingly.

We have now characterized completely the general model we are looking for. Every theory of gravity coupled with a scalar described by the action (11), will allow in the non-relativistic, static, weak-field limit for a solution

$$
\Phi=C \ln \frac{r}{L}-\frac{G M}{r}+\mathcal{O}\left(r^{-2}\right)
$$

if the coupling function satisfies Eqs. (77),(10). For instance a simple solution of Eqs. (7), (10) is

$$
H=\frac{C}{2} \ln \left(1+\frac{r^{2}}{L^{2}}\right) .
$$

The form of the potential $V(\varphi)$ is only constrained by Eq. (6), which is necessary for the consistency of the non-relativistic, weak-field approximation. An other physical condition that has to be imposed on the form of the potential is stability, i.e. $V(\varphi)$ must be bounded from below and eventually it must allow for a local minimum. Excitations near this minimum must have enough heavy mass to be compatible with particle physics phenomenology.

\section{Scalar field with a power-law potential}

As a simple example and illustration of our general approach we consider the case of a scalar field with a power-law potential

$$
V=N L^{-4}(\varphi L)^{(2-2 / \alpha)}
$$


where $N$ and $\alpha$ are arbitrary dimensionless parameters. The weak-field equations (4) for $\varphi$ are readily solved to give

$$
\varphi=D L^{-1}\left(\frac{r}{L}\right)^{\alpha}
$$

where $D=\left([N(\alpha-1)] /\left[\alpha^{2}(\alpha+1)\right]^{\alpha / 2}\right.$. Using solution (14) one easily find that the consistency conditions (6) are satisfied if $\alpha<-1$.

The coupling function $H$ is constrained by Eqs. (17),(10). A simple solutions is given by Eq. (12), which in view of Eq. (14) becomes:

$$
H(\varphi)=\frac{C}{2} \ln \left[1+\left(\frac{L \varphi}{D}\right)^{\frac{2}{\alpha}}\right] .
$$

For $\alpha<-1$ the potential $V(\varphi)$ is bounded from below in the range $0<\varphi<\infty$, corresponding to the physical range $0<r<\infty$ of the radial coordinate $r$. Thus the system is stable and in principle one can also deform the potential in such a way that $V(\varphi)$ has

local minimum at $\varphi=\varphi_{0}$, with $V^{\prime \prime}\left(\varphi_{0}\right)=m_{\varphi}^{2}$. The parameters in the potential have to be chosen in such a way that the mass $m_{\varphi}$ of the local excitation is heavy enough to be compatible with the experimental results about the absence of long range scalar forces.

\section{$6 \quad$ Large-distance modifications of gravity}

The gravity model (1) can be used both as an effective description of infrared modification of gravity, e.g. in the brane world scenario, or also as an Einstein-like description of Newtonian modified dynamics.

In the first case the scalar field $\varphi$ could represent an effective $4 \mathrm{D}$ parametrization of those effects that modifies gravity at large distances ( for instance the embedding of our 3-brane in a higher dimensional world). Apart from the potential $V(\varphi)$, which does not determine the infrared modifications of gravity, the model is parametrized by a coupling function $H$ which depends on a length scale $L$ and a coupling constant $C$. $H$ is zero together with its first derivatives at $r=0$ and stays almost constant (and vanishing) for $r \ll L$. In this regime the gravitational potential is given by the Newtonian expression and the theory reduces to General relativity. For $r \gg L, H \sim \ln (r / L)$, the gravitational potential is dominated by a $\ln r$ term and the theory deviates from general relativity, still giving an effective metric description of the gravitational interaction.

In the second case, one can use our model to explain the rotation curves of the galaxies without postulating the presence of dark matter. To this end one introduces a constant acceleration $a_{0} \sim 10^{-29} \mathrm{~cm}$ such that for $a \gg a_{0}$ one recovers standard Newtonian dynamics. In fact, in this case $\sqrt{G M} / r \gg a_{0}$ and the Newtonian term in the potential (11) dominates over the logarithmic term. On the other hand for $a \sim a_{0}$ the leading logarithmic term dominates the gravitational potential (11) and the rotation curves of the galaxies can be explain using the gravitational potential (11) and identifying the constant $C$ as

$$
C=\left(\frac{M}{\sigma}\right)^{1 / 2}
$$

where $M$ is the mass of the galaxy and $\sigma$ is an universal constant, which can be determined using the Tully-Fisher law [15, 14]. 


\section{Jordan frame and equivalence with $f(R)$ theories}

The gravitational model described by the action (1) is equivalent to a scalar-tensor theory of gravity. It is a scalar-tensor theory of gravity written in the Einstein frame. The transition from the Einstein to the Jordan frame is given by the Weyl transformation,

$$
g_{\mu \nu}=e^{-2 H} \hat{g}_{\mu \nu}
$$

where $\hat{g}_{\mu \nu}$ is the metric in the Jordan frame. In this frame the action (1) becomes

$$
\begin{aligned}
S & =\int d^{4} x \sqrt{-\hat{g}} e^{-2 H}\left[\frac{1}{16 \pi G} R(\hat{g})+\left(\frac{3}{8 \pi G} H^{\prime}-1\right) \partial_{\nu} \varphi \partial^{\nu} \varphi-e^{-2 H} V\right]+ \\
& -\left(M \int d s_{M}+m \int d s_{m}\right) .
\end{aligned}
$$

In the Einstein frame theories described by the action (1) lead to a breakdown of the equivalence principle at galactic scales [14]. Conversely in the Jordan frame the particles do not couple to the scalar field. The geodesic equation depends only on the metric $\hat{g}_{\mu \nu}$. On the other hand in the Jordan frame the theory has the usual weaknesses of scalartensors theories of gravity: Newton constant depends on the coordinates and light bending is not correctly reproduced (see e.g. Ref. [6]).

One can also consider the weak-field limit of the field equations stemming from the action (18) along the lines described in the previous sections. Because now the geodesic

equation for the test particle depends only on the metric $\hat{g}_{\mu \nu}$, we set $\hat{g}_{\mu \nu}=\eta_{\mu \nu}+\hat{h}_{\mu \nu}$ and $\Phi=-\hat{h}_{00} / 2$. In the non-relativistic, weak-field limit we can approximate $e^{ \pm 2 H}=1 \pm 2 H$ and the equation of motion for $\Phi, \varphi$ and the geodesic equation coincide with (4) upon use of Eqs. (6), (77). Hence the solutions (10),(11), (12) found in Sect. 4 are also solution of the theory in the Jordan frame.

It is well known that gravity models whose Lagrangians are given by functions of the scalar curvature $f(R)$ are on-shell equivalent to Einstein gravity coupled to a scalar field (see e.g. Ref. 6]). General models may also involve a an arbitrary coupling function $e^{H(\varphi)}$ [6] and be therefore equivalent to the model described by the action (1). The potential $V(\varphi)$ for the scalar field is determined by the the function $f(R)$. Our characterization of gravity models with non-Newtonian weak-field limit can be therefore easily extended to the case of gravity theories with $f(R)$ Lagrangians.

The existence of non-Newtonian solutions seems a quite generic feature of $f(R)$ theories. In fact the potential $V$ is determined entirely by the function $f$ and the requirement for the existence of non-Newtonian solutions constrain very weakly the form of $V$. Also in this case the existence of the non-Newtonian weak-field limit is related to the presence of a non trivial coupling function $e^{H}$.

\section{Conclusion}

In this paper we have show that the existence of a non-Newtonian weak-field limit in which the gravitational potential has a large distance $\ln (r / L)$ leading term is a quite generic feature of a broad class of models of Einstein gravity coupled with a scalar field and of theories of gravity with an $f(R)$ lagrangian, when a scalar field-dependent matter 
coupling function is introduced. This class of models has been selected by enforcing rather general physical conditions on the form of the non-relativistic gravitational potential.

The gravity models proposed and investigated in this paper may be very useful to give an effective description for large-distance deviations of the gravitational interaction from its GR behavior, e.g in brane-world scenarios. Another interesting field of application is their use to describe modifications of Newtonian dynamics at small accelerations, e.g. for explaining rotation curves of galaxies.

The most sensible and critical point of our approach is the physical origin of the coupling function $e^{H(\varphi)}$. Its presence is quite natural in scalar-tensor theories of gravity, e.g. Brans-Dicke theory and related to the choice of what may be called the physical Weyl frame [16, 17]. But its physical interpretation is far from being clear. One possibility is that it gives a local effective description of non-local effects. For instance, in brane-world scenarios it may be considered as a local parametrization of nonlocal effects generated by the embedding of the 3D brane in the higher dimensional spacetime.

\section{References}

[1] G. R. Dvali, G. Gabadadze and M. Porrati, Phys. Lett. B 485 (2000) 208 arXiv:hep-th/0005016.

[2] N. Arkani-Hamed, H. C. Cheng, M. A. Luty and S. Mukohyama, gravity," JHEP 0405 (2004) 074 arXiv:hep-th/0312099.

[3] M. Milgrom, Astrophys. J. 270 (1983) 365.

[4] M. Milgrom, Annals Phys. 229 (1994) 384 arXiv:astro-ph/9303012.

[5] J. A. de Diego, arXiv:0807.0617 [physics.space-ph].

[6] J. P. Bruneton and G. Esposito-Farese, Phys. Rev. D 76 (2007) 124012 [Erratum-ibid. D 76 (2007) 129902] arXiv:0705.4043 [gr-qc]].

[7] B. Famaey, G. Gentile, J. P. Bruneton and H. S. Zhao, Phys. Rev. D 75 (2007) 063002 arXiv:astro-ph/0611132.

[8] K. Trencevski and E. G. Celakoska, arXiv:gr-qc/0604068.

[9] O. Bertolami and J. Paramos, arXiv:gr-qc/0411020.

[10] S. Capozziello, V. F. Cardone and A. Troisi, Mon. Not. Roy. Astron. Soc. 375, 1423 (2007) arXiv:astro-ph/0603522.

[11] V. Trimble, Ann. Rev. Astron. Astrophys. 25 (1987) 425.

[12] M. Kunz and D. Sapone, Phys. Rev. Lett. 98 (2007) 121301 arXiv:astro-ph/0612452.

[13] M. Cadoni and P. Pani, arXiv:0812.3010 [hep-th].

[14] M. Cadoni, Gen. Rel. Grav. 36 (2004) 2681 arXiv:gr-qc/0312054.

[15] R. B. Tully and J. R. Fisher, Astron. Astrophys. 54 (1977) 661. 
[16] V. Faraoni and E. Gunzig, Int. J. Theor. Phys. 38 (1999) 217 arXiv:astro-ph/9910176.

[17] S. Capozziello, R. de Ritis and A. A. Marino, Class. Quant. Grav. 14 (1997) 3243 arXiv:gr-qc/9612053|. 